\title{
Numerische Modellierung mit frei verfügbarer Software
}

Numerische Modellierung ist bereits seit Jahrzehnten fixer Bestandteil in vielen naturwissenschaftlichen und technischen Disziplinen. Numerische Modelle zur Berechnung von hydrodynamischen Prozessen, vom hydrologischen Niederschlags-Abflussmodell bis zum dreidimensionalen Navier-StokesGleichungslöser (Computational Fluid Dynamics - CFD), bilden heutzutage als Stand der Technik die Grundlage unter anderem für die Planung von wasserbaulichen Anlagen, für ökologische Untersuchungen sowie als Entscheidungswerkzeug für wasserwirtschaftliche Fragestellungen. Man denke an die österreichweiten Abflussuntersuchungen, welche als Grundlage für Überflutungs- sowie Gefahrenzonenkarten und weiterführend für die Erarbeitung von Risikomanagementkonzepten dienen.

Die Gewässer als Lebens- und Kulturräume zu verstehen spiegelt sich auch in den Modellierungsansätzen wider, indem zunehmend versucht wird, den Wasserkreislauf und sozio-ökonomische Aspekte modelltechnisch gesamtheitlich zu erfassen. Entwicklungen wie diese gehen meistens von öffentlichen Institutionen und Forschungseinrichtungen aus und werden in Fachartikeln präsentiert. Häufig werden dabei neue Modellansätze als Open-Source-Software der Allgemeinheit zur Verfügung gestellt. Die Forschungsergebnisse können wiederum die Grundlage für die Entstehung neuer oder die Weiterentwicklung kommerziell vermarktbarer Software bilden.

Für all diese Fragstellungen kommen (hydronumerische) Modelle zum Einsatz, die verschiedenen Lizenzierungen unterliegen. Die Lizenzierung von Software ist ein kompliziertes und oft schwer durchschaubares Feld, wobei unterschiedlichste Arten der Vermarktung möglich sind. Wir möchten Ihnen hier vorab einen groben Überblick darüber geben.

Kommerzielle, proprietäre Modellierungssoftware ist in der wasserbaulichen Ingenieurspraxis wohl die am häufigsten eingesetzte Softwareart. Typischerweise ist diese sehr gut validiert, schnell zu erlernen, benutzerfreundlich zu bedienen und für konventionelle Aufgabenstellungen gut geeignet. Diese wesentlichen Eigenschaften und der vielfach angebotene Support als Dienstleistung für die Benutzung haben natürlich ihren Preis. Als Nachteil kommerzieller Software würden wir vor allem die Tatsache sehen, dass die Programme üblicherweise als "black box" vertrieben werden, das heißt, man kann nicht einfach eruieren und nachvollziehen, wie die Software eine jeweilige Fragestellung löst. Des Weiteren ist es auch nicht möglich, aktiv in den Code einzugreifen und Adaptierungen daran vorzunehmen.

Als Freeware bezeichnet man proprietäre Software, die den Benutzerinnen und Benutzern kostenlos zur Verfügung gestellt wird, die Entwicklung aber dem Anbieter vorbehalten ist.

Open-Source-Software ist frei verfügbar und der Quellcode von Open-Source-Software öffentlich zugänglich. Für Open-Source-Software existieren grundsätzlich verschiedene Lizenzmodelle, welche unter anderem die Nutzung, kommerzielle Verwertung oder z. B. auch die Zitiervorschrift der Urheberschaft regeln. Aus wissenschaftlicher Sicht eröffnet Open-Source-Software den Anwenderinnen und Anwendern die Möglichkeit, in den Quellcode zu blicken und somit die modellierten Vorgänge aus der Natur bzw. die Modellierung technischer Maßnahmen und Konstruktionen nachvollziehen zu können sowie einen bestehenden Quellcode für eigene Forschungszwecke zu adaptieren bzw. zu verbessern. Häufig sind sehr aktive „Usergroups“ bei diesem Softwaretyp anzutreffen (z. B. der Telemac User Club mit seinem Forum, Delft Software Days). Gerade dies ist ein großer Vorteil in naturwissenschaftlichen und technischen Disziplinen, da damit eine aktive Weiterentwicklung und Anpassung der Softwarepakete an die Anforderungen der Benutzer und Benutzerinnen gegeben ist. Als Nachteil dieses Softwaretyps kann sich, salopp formuliert, die hin und wieder schwierigere Bedienung der Programme herausstellen, die des Öfteren auch schon mit der Installation beginnen kann. 
Frei verfügbare Software subsumiert kostenlos verfügbare Open-Source-Programme sowie FreewareProdukte. Dieses Heft beinhaltet sechs Beiträge ausgewählter Anwendungen von frei verfügbarer Software für hydrologische, hydrodynamische sowie strukturmechanische Fragestellungen.

Gegenleithner et al. geben zu Beginn eine Einführung in Open-Source-Software und Freeware sowie einen kurzen historischen Abriss der Softwareentwicklung mit dem Fokus auf wasserbauliche Fragstellungen, wobei die Rollen des Entwicklers und der Entwicklerin sowie des Anwendenden im Laufe der Zeit beleuchtet werden. Die Autoren gehen des Weiteren auf die häufig geführte Diskussion über Vor- und Nachteile von kostenpflichtiger und frei verfügbarer Software ein. Sie weisen darauf hin, dass letztendlich die Wahl der geeigneten Modellierungssoftware je nach Aufgabenstellung sowie Kenntnissen der Anwenderinnen und Anwender erfolgen muss.

In vier kurz gehaltenen Anwendungsbeispielen werden der Einsatz von Open-Source-Software und beispielhafte Quellcode-Adaptierungen für Forschungszwecke als auch für industrielle Aufgabenstellungen gezeigt. Im ersten Beispiel wird die Implementierung von Ansätzen zur Berechnung von hydraulischen Verlusten bei Brücken im tiefengemittelten 2D numerischen Modell Telemac-2D präsentiert. Die Modellierung von instationären Strömungsvorgängen in Stauketten, beeinflusst durch Kraftwerkssteuerungen, wird am zweiten Beispiel als Implementierung von internen Randbedingungen in das 2D numerische Modell Telemac-2D gezeigt. Hoch komplexe Modellkopplungen zwischen meteorologischen, hydrologischen und hydrodynamischen Open-Source-Modellen, vorgestellt im dritten Beispiel, ermöglichen Echtzeithochwasservorhersagen inklusive der Bestimmung von Echtzeitüberflutungsflächen. Schlussendlich wird als weiteres Beispiel der Einsatz von Open-Source-Produkten für die Analyse von komplexen Datenmengen präsentiert.

Die bekannte, von der ETH Zürich entwickelte, hydronumerische Modellierungssoftware BASEMENT wird von Vetsch et al. vorgestellt. Das Programm besteht aus einem eigenständigen 1D- sowie einem 2D-Modul, die miteinander koppelbar sind. Mithilfe der Software ist des Weiteren die Berechnung von Feststofftransportprozessen möglich. Im vorliegenden Beitrag liegt ein Fokus auf der Beschreibung der aktuellen Entwicklung der Software hinsichtlich Rechengeschwindigkeit. So ist die neueste BASEMENT-Version auf Grafikkarten lauffähig, womit ein deutlicher Performanzgewinn erzielt werden kann. Vetsch et al. zeigen darüber hinaus eine breite Palette von Anwendungsbeispielen, beginnend bei Stauregelungsberechnungen über Geschiebebewirtschaftungsfragen bis hin zum Einsatz des Programms für ökohydraulische Aufgabenstellungen.

Eine sehr ausführliche Beschreibung der Anwendung von 3D CFD Open-Source-Software anhand des Programms OpenFOAM geben Shahriari et al. Mittels vier Beispielen wird der Einsatz dieser Software, vornehmlich für Forschungszwecke, hervorgehoben. Ein neues in die Software implementiertes Schema für die Zeitintegration wird präsentiert, welches, bei deutlichem Performanzgewinn, genauere Berechnungen in Large-Eddy-Simulationen (LES) ermöglicht. In akademischen Beispielen werden verschiedene Ansätze für die Turbulenzmodellierung verglichen. Ein Brückenschlag zur industriellen Anwendung der CFD-Software OpenFOAM wird anhand der Modellierung der Zuströmung zu einem Laufkraftwerk vorgestellt.

Peßenteiner et al. zeigen in ihrem Beitrag den Einsatz frei verfügbarer Software für Untersuchungen im Wildbachbereich. Dabei werden, basierend auf diversen erhobenen Messdaten, Modellkopplungen präsentiert, beginnend mit dem Niederschlags-/Abflussmodell WaSiM bis hin zum hydrodynamischen Modell Telemac-2D und dem Sedimenttransportmodell SISYPHE. Im Beitrag werden erste Ergebnisse dieses Ansatzes für die Anwendung am Schöttlbach in der Obersteiermark gezeigt. Die vielversprechenden Ergebnisse lassen erhebliche Verbesserungen im Verständnis der Sedimentkaskade in Wildbacheinzugsgebieten erwarten.

Das Open-Source Stormwater Management Model (SWMM) für die hydrologisch-hydraulische Kanalnetzmodellierung wird von Krebs vorgestellt. Das Modell und die Nachbearbeitung der Ergebnisse 


\title{
Editorial
}

bzw. deren Analyse mittels des Open-Source geografischen Informationssystems QGIS und der OpenSource Skript- (Programmier-) sprachen R und Python wird am Anwendungsbeispiel der Stadt Lahti (Finnland) anschaulich erläutert.

Freeware wie CADAM2D ermöglichen dem Ingenieur/der Ingenieurin, geometrische und mechanische Zusammenhänge bei Talsperren relativ einfach und daher vor allem in frühen Projektstadien zu berechnen. Staudacher und Zenz beschreiben in ihrem Beitrag die Anwendung des Programms CADAM2D, welches die Spannungsverteilung der Aufstandsfläche bzw. der Arbeitsfugen des Sperrenkörpers unter Berücksichtigung der Balkentheorie ermittelt und die relevanten Sicherheitsfaktoren wie z. B. Gleiten oder Kippen ausgibt.

In den Beiträgen dieser Ausgabe der Österreichischen Wasser- und Abfallwirtschaft können nur einige Möglichkeiten der Modellierung mit frei verfügbarer Software vorgestellt werden. Anwenderinnen und Anwender können mittlerweile im Internet aus einer breiten Palette frei verfügbarer Software für wasserwirtschaftliche Aufgabenstellungen wählen.

Welche Art von Modell man schlussendlich wählt, hängt von verschiedenen Faktoren ab. Für die Bearbeitung einer konkreten Aufgabenstellung sind der Anwender und die Anwenderin von kommerzieller Software grundsätzlich von den implementierten Optionen abhängig. Im Fall der Wahl einer Open-Source-Software mag im ersten Augenblick mehr Flexibilität vorhanden sein, jedoch steigt auch die Komplexität während des Anwendens. So sind es vordergründige, pragmatische Fragen für den Entscheidungsprozess: Wähle ich eine Software, welche einfach bedienbar ist und für die meisten Aufgabenstellungen ausreicht oder ein Produkt für Forschungszwecke und die Anwendung in „Nischenprojekten“ von heute und Standardprojekten von morgen, weil sie projektspezifisch adaptierbar ist? Darüber hinaus stellt sich die Frage, ob die Kosten für die Software relevant sind oder ob dem Anwender/der Anwenderin schlicht und einfach die Modellauswahl seitens eines Auftraggebers vorgeschrieben wird?

\author{
Assoc. Prof. DI Dr. J. Schneider (四) \\ Institut für Wasserbau und Wasserwirt- \\ schaft, Technische Universität Graz, \\ Stremayrgasse 10 , \\ 8010 Graz, Österreich \\ schneider@tugraz.at
}

\section{Dr. C. Dorfmann}

flow engineering, Ingenieurbüro für

Kulturtechnik und Wasserwirtschaft,

Brockmanngasse 108,

8010 Graz, Österreich

clemens.dorfmann@flowengineering.at 